DOI: $10.4046 / \operatorname{trd} 2011,70.2 .139$

ISSN: 1738-3536(Print)/2005-6184(Online)

Tuberc Respir Dis 2011;70:139-142

Copyright(C)2011. The Korean Academy of Tuberculosis and Respiratory Diseases. All rights reserved.

\title{
Cutaneous Metastasis from Lung Cancer: A Single-Institution Retrospective Analysis
}

\author{
Jong Hwan Lee, M.D., Se Jin Ahn, M.D., Hyung Jin Kim, M.D., Sang Eon Jang, M.D., Geum Youb Noh, \\ M.D., Hye-Ryoun Kim, M.D., Cheol Hyeon Kim, M.D., Jae Cheol Lee, M.D., Ph.D. \\ Department of Internal Medicine, Korea Cancer Center Hospital, Korean Institute of Radiological \& Medical Sciences, Seoul, \\ Korea
}

Background: Lung cancer is responsible for substantial proportions of cutaneous metastasis from internal malignancies. The aim of this study was to evaluate the clinical manifestations and outcomes of cutaneous metastasis in Korean lung cancer patients.

Methods: On a retrospective basis, we analyzed medical records of all patients diagnosed with lung cancer from 2000 to 2006.

Results: Cutaneous metastases were found in 10 of 4,385 patients. The number of cases was highest for squamous cell carcinoma. However, there was no metastasis from 754 cases of small cell carcinomas. Cutaneous metastasis was detected during staging work-up in 4 patients and it was the presenting sign of recurrence post-operative in 2 patients. Average time from the diagnosis to discovery of cutaneous metastasis was 16.3 months and median survival was 8.5 months (range, 1.8 19.1 months).

Conclusion: Physicians should be acquainted with clinical manifestations and outcomes of cutaneous metastasis from lung cancer to detect new, recurrent cancer, or disease progression, and to administer appropriate and prompt management.

Key Words: Lung neoplasms; Neoplasm Metastasis; Skin neoplasms

\section{Introduction}

Cutaneous metastasis from visceral malignancies is not frequent and this represents only about $2 \%$ of all skin tumors ${ }^{1,2}$. The incidence of cutaneous metastasis from visceral malignancies has been estimated to be 2 $9 \%{ }^{3,4}$. The most common cancers to metastasize to the skin differ according to the patients' gender. In women, the majority of cutaneous metastases develop from breast cancer, followed by colon, lung and ovary cancers, while lung, colon and head and neck cancers are the most common cancers to display cutaneous meta-

Address for correspondence: Jae Cheol Lee, M.D., Ph.D. Department of Internal Medicine, Korea Cancer Center Hospital, Korea Institute of Radiological \& Medical Sciences, 215-4, Gongneung-dong, Nowon-gu, Seoul 139-706, Korea Phone: 82-2-970-1206, Fax: 82-2-970-2438

E-mail: jclee@kcch.re.kr

Received: Aug. 17, 2010

Accepted: Oct. 1, 2010 stases in men ${ }^{5}$. Although cutaneous metastasis of lung cancer is relatively less common than that to other organs such as the brain, bones, liver and adrenal glands, lung cancer is responsible for a substantial proportion of the cutaneous metastasis from internal malignancies ${ }^{6}$. Cutaneous metastasis can often be a presenting manifestation or a signal to indicate disease progression. Therefore, careful examination of a skin lesion is required for physicians to detect new or recurred cancer or to recognize the proper time to re-start treatment for stable lung cancer patients who are being followed up. Considering the possible different manifestations and outcomes according to race, this study aimed to report on the cutaneous metastases from lung cancer that were seen at a tertiary referral cancer center in Korea. 


\section{Materials and Methods}

We retrospectively reviewed all the patients who were diagnosed with lung cancer at Korea Cancer Center Hospital during the period between 2000 and 2006. We found 4,385 patients with pathologically proven lung cancer via a review of the medical records, and cutaneous metastasis developed in ten of them. We analyzed their clinical features, including age, gender, location, the number and type of metastases, the presence of metastases to other organs and the prognosis. The Ethical Review Committee of Korea Cancer Center Hospital approved the protocol for this study.

\section{Results}

Cutaneous metastasis from lung cancer was confirmed in ten patients $(0.2 \%)$ among the 4,385 patients. The median age of these patients ( 8 men and 2 women) was 66 years (age range, 51 85 years). Eight patients were smokers (range, 30 55 pack years). The histological patterns of cutaneous metastasis were as follows; squamous cell carcinoma (6 patients, 0.42\%), adenocarinoma (3 patients, 0.19\%) and large cell neuroendocrine carcinoma (1 patient, 1.6\%). Interestingly, there was no cutaneous metastasis from the 754 cases of small cell carcinomas.

Regarding the number of skin metastases, 7 patients possessed a single location while others had 2 or 3 locations. The most common site was the scalp, and this was followed by the anterior chest wall. Most lesions presented as single or multiple nodules that were relatively firm and hemispherical-shaped.

Cutaneous metastasis was detected during the staging work-up for lung cancer in 4 patients (patients 1, 4, 7 and 9). The average time from the diagnosis of lung cancer to the discovery of cutaneous metastasis was 16.3 months (range, 1.1 60.8 months) in the other patients. All the patients with cutaneous metastasis died and the median survival was 8.5 months (range, 1.8 19.1 months).

Table 1 summarized the data about the demographic characteristics, the clinical manifestations and the outcomes.

\section{Discussion}

The reported incidence of cutaneous metastasis from lung cancer has ranged from 1 to $12 \%{ }^{7-12}$ whereas it was $0.23 \%$ in our study, and this is less than that of the previous reports. It is unlikely that a cutaneous metastasis is missed during follow-up because most patients can easily recognize this and they probably report it to their physician. Although the lower incidence rate in this study might be related with some limitations of retrospective analysis such as follow-up loss and improper medical recording, recent progress in diagnostic and therapeutic modality including wide use of EGFR tyrosine kinase inhibitors would contribute to this difference because most of previous studies enrolled patients in the 1980s or 1990s requiring further investigations on this matter.

A skin lesion may sometimes herald internal malignancy as Brownstein and Helwig found that this is frequently the case for lung and kidney tumors ${ }^{13}$. Skin-related symptoms were the chief complaints in about 20 $\sim 40 \%$ of the patients with metastatic skin cancer ${ }^{7,13}$. In our study, cutaneous metastasis was detected at the time of diagnosing lung cancer in 4 patients, although all of them were accompanied by other sites of metastasis. However, only one patient complained of skin problems because the dermal symptoms of the other patients were negligible compared with the symptoms from the other organs with metastasis. Nodular skin lesion was the presenting sign of recurred lung cancer after operation in two patients. Subsequent brain metastasis developed in both these patients. This clearly showed that new skin lesions in lung cancer patients should be carefully examined to detect early recurrence or aggravated disease.

The skin was the only metastatic site in two patients and it was a single lesion in one of them. There have been some reports demonstrating a good prognosis after an operation for lung cancer and the isolated metastasis 
Tuberculosis and Respiratory Diseases Vol. 70. No. 2, Feb. 2011

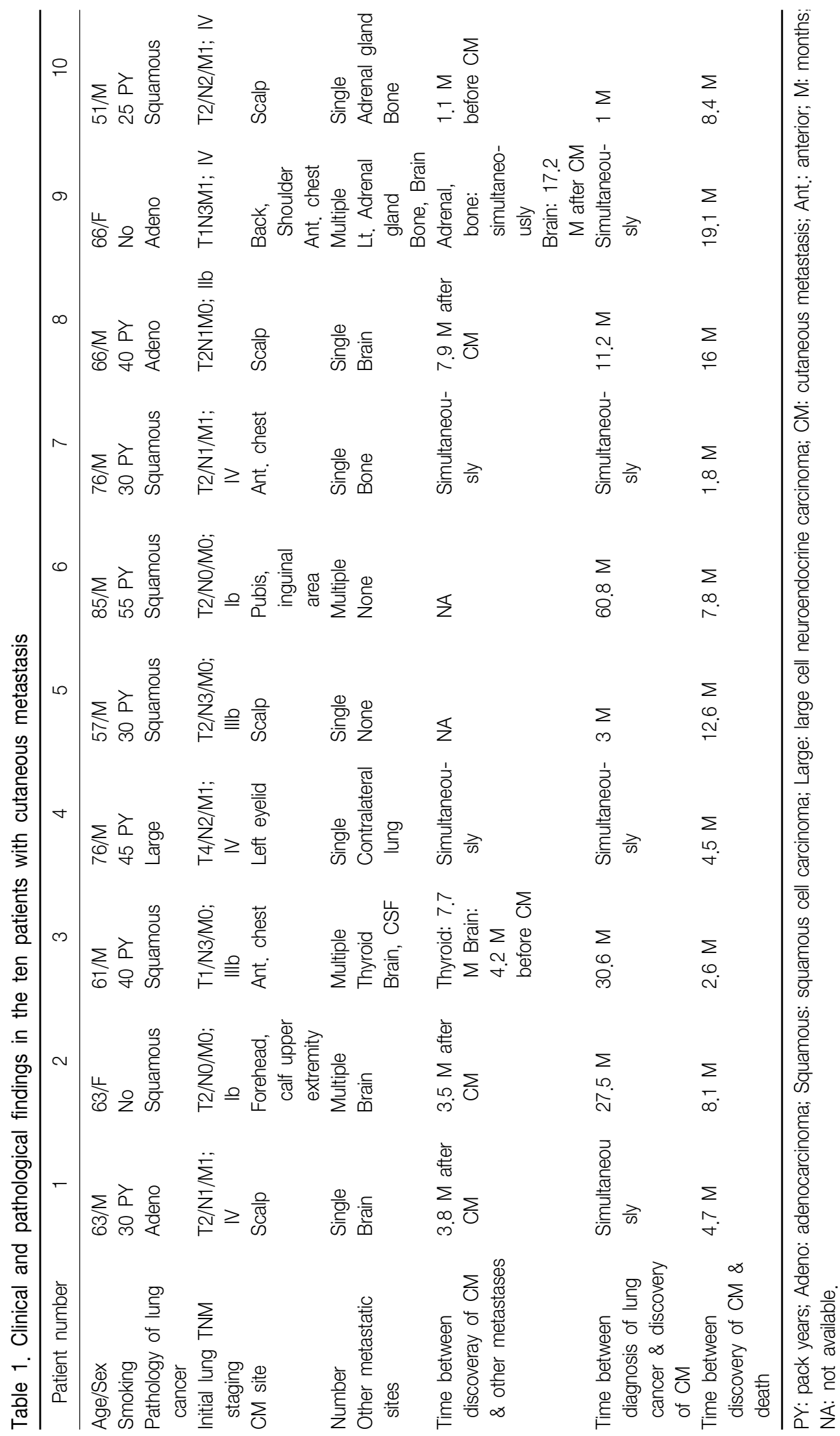


to brain ${ }^{14}$, adrenal ${ }^{15}$, small bowel ${ }^{16}$ etc., although we could not find any report of a long-term survivor after removal of lung cancer and skin metastasis. For our cases, we did not attempt surgery for the metastasis because the chance of cure seemed to be low considering the advanced initial lung cancer stage (IIIb).

Terashima and Kanazawa ${ }^{7}$ showed that adenocarcinoma was the most prevalent histologic type of cutaneous metastasis ${ }^{8}$ while large cell carcinoma was reported by Hidaka et al. ${ }^{6}$ to have the highest rate of metastasis to skin, as was also noted in our study. One of the distinguishing features regarding the histology in most of the studies, including ours, is that the metastasis rate of small cell carcinoma is very low. Considering that small cell carcinoma can easily and early metastasize to various organs, this low metastasis rate of small cell carcinoma seems to be peculiar and the reason for it should be further explored.

The most common site of the cutaneous metastasis was the scalp because of the rich blood flow, and the incidence rate noted by Brownstein and Helwig was $54 \%{ }^{7}$, although in some reports, the most common site of the cutaneous metastasis was the anterior part of the chest or back ${ }^{3,6}$. Our result showing that four patients had scalp metastasis was comparable to this finding.

Hidaka et al. ${ }^{6}$ reported that the median survival time after skin metastasis was 4 months. The mean survival time from the time of the diagnosis of skin metastasis was 4.9 months in a study by Terashima et al. indicating a poor prognosis ${ }^{7}$. However, our study showed a better median survival period of 8.5 months, which might reflect the recent advances of chemotherapeutic agents and supportive care.

In conclusion, physicians should be acquainted with clinical manifestations and outcomes of skin metastasis to detect new or recurred cancer or disease progression and to administer the appropriate and prompt management for patients suffering with lung cancer.

\section{References}

1. Nashan D, Müller ML, Braun-Falco M, Reichenberger
S, Szeimies RM, Bruckner-Tuderman L. Cutaneous metastases of visceral tumours: a review. J Cancer Res Clin Oncol 2009;135:1-14.

2. Hu SC, Chen GS, Wu CS, Chai CY, Chen WT, Lan CC. Rates of cutaneous metastases from different internal malignancies: experience from a Taiwanese medical center. J Am Acad Dermatol 2009;60:379-87.

3. Krathen RA, Orengo IF, Rosen T. Cutaneous metastasis: a meta-analysis of data. South Med J 2003;96:164-7.

4. Hu SC, Chen GS, Lu YW, Wu CS, Lan CC. Cutaneous metastases from different internal malignancies: a clinical and prognostic appraisal. J Eur Acad Dermatol Venereol 2008;22:735-40.

5. Mueller TJ, Wu H, Greenberg RE, Hudes G, Topham $\mathrm{N}$, Lessin SR, et al. Cutaneous metastases from genitourinary malignancies. Urology 2004;63:1021-6.

6. Hidaka T, Ishii Y, Kitamura S. Clinical features of skin metastasis from lung cancer. Intern Med 1996;35:45962.

7. Terashima T, Kanazawa M. Lung cancer with skin metastasis. Chest 1994;106:1448-50.

8. Kamble R, Kumar L, Kochupillai V, Sharma A, Sandhoo MS, Mohanti BK. Cutaneous metastases of lung cancer. Postgrad Med J 1995;71:741-3.

9. Perng DW, Chen CH, Lee YC, Perng RP. Cutaneous metastasis of lung cancer: an ominous prognostic sign. Zhonghua Yi Xue Za Zhi (Taipei) 1996;57:343-7.

10. Rubinstein RY, Baredes S, Caputo J, Galati L, Schwartz RA. Cutaneous metastatic lung cancer: literature review and report of a tumor on the nose from a large cell undifferentiated carcinoma. Ear Nose Throat J 2000; 79:96-7, 100-1.

11. Barbetakis N, Samanidis G, Paliouras D, Samanidou E, Tzimorota Z, Asteriou C, et al. Facial skin metastasis due to small-cell lung cancer: a case report. J Med Case Reports 2009;3:32.

12. Coslett LM, Katlic MR. Lung cancer with skin metastasis. Chest 1990;97:757-9.

13. Brownstein MH, Helwig EB. Spread of tumors to the skin. Arch Dermatol 1973;107:80-6.

14. Chee RJ, Bydder S, Cameron F. Prolonged survival after resection and radiotherapy for solitary brain metastases from non-small-cell lung cancer. Australas Radiol 2007; 51:186-9.

15. Mercier O, Fadel E, de Perrot M, Mussot S, Stella F, Chapelier A, et al. Surgical treatment of solitary adrenal metastasis from non-small cell lung cancer. J Thorac Cardiovasc Surg 2005;130:136-40.

16. Kim MS, Kook EH, Ahn SH, Jeon SY, Yoon JH, Han MS, et al. Gastrointestinal metastasis of lung cancer with special emphasis on a long-term survivor after operation. J Cancer Res Clin Oncol 2009;135:297-301. 